\title{
Impacto de un programa de control de la calidad de la prescripción de antibióticos en un hospital de La Habana, Cuba
}

\author{
Humberto Guanche Garcell, ${ }^{1}$ Juan José Pisonero Socias, ${ }^{1}$ \\ Raimy Enseñat Sánchez, ${ }^{1}$ Irene Fiterre Lancis, ${ }^{1}$ \\ loanna Mir Narbona, ${ }^{1}$ Belkis García Arzola ${ }^{1}$ \\ Gilberto Pardo Gómez ${ }^{1}$ y Francisco Gutiérrez García ${ }^{2}$
}

Forma de citar

Guanche Garcell H, Pisonero Socias JJ, Enseñat Sánchez R, Fiterre Lancis I, Mir Narbona I, García Arzola B, et al. Impacto de un programa de control de la calidad de la prescripción de antibióticos en un hospital de La Habana, Cuba. Rev Panam Salud Publica. 2011;30(6):598-602.

RESUMEN Objetivo. Demostrar la eficacia de un programa de control de la calidad de la prescripción de antibióticos en el Hospital Docente Clínico Quirúrgico Joaquín Albarrán de La Habana, Cuba. Métodos. Estudio de intervención realizado en el período del 1 de mayo de 2008 a 31 de marzo de 2011, que incluyó la evaluación de la calidad de la prescripción y la retroalimentación de la información, actividades educativas, funcionamiento de un comité de antibióticos y la elaboración de protocolos de uso de antimicrobianos. Se construyó un gráfico aritmético simple de la serie temporal y se compararon los valores absolutos de las proporciones de la serie entre sí. Para comprobar la existencia de tendencia en la serie se ajustó a un modelo de regresión lineal simple.

Resultados. Se evaluaron las prescripciones de antibióticos de 2941 pacientes, en las que se observó una serie irregular, con proporción de uso inadecuado entre 48,4\% y 30,7\% en los primeros tres meses analizados. Se encontró que el valor tomado por la pendiente de regresión, aunque se encontraba cercano a cero, era negativo y significativamente diferente de cero $(\beta=$ $-0,29 ; \mathrm{P}=0,02)$.

Conclusiones. El programa de control de antibióticos mejoró la calidad de la prescripción a los pacientes hospitalizados.

Palabras clave Prescripción de medicamentos; control de medicamentos y narcóticos; agentes antibacterianos; Cuba.

Las bacterias han demostrado tener la capacidad de vencer las habilidades humanas para descubrir y utilizar antibióticos mediante el desarrollo de la resistencia microbiana. Esta, a su vez, ha adquirido carácter pandémico y actualmente abarca a todas las bacterias,

\footnotetext{
Hospital Joaquín Albarrán, La Habana, Cuba. La correspondencia se debe dirigir a Humberto Guanche Garcell. Correo electrónico: guanche@infomed.sld.cu 2 Instituto Nacional de Nefrología, La Habana,Cuba.
}

regiones y países del mundo. Desde principios de este siglo, en consideración a su impacto en la calidad y eficiencia de los sistemas de salud, algunas organizaciones internacionales emitieron recomendaciones para controlar la resistencia a los antibióticos (1).

Se ha demostrado fehacientemente que la aparición de resistencia a los antibióticos ha estado condicionada por la presión selectiva en la flora microbiana, que tiene como un elemento esencial el uso inadecuado de los antibióticos por los profesionales de la salud (2-6). Un estudio nacional realizado en Cuba para determinar la prevalencia puntual de la infección nosocomial mostró que 20,6\% de los pacientes internados en hospitales y sin evidencia de infección habían utilizado antimicrobianos (7). Asimismo, en estudios realizados en servicios clínicos y quirúrgicos de dos hospitales, se en- 
contraron frecuencias de administración inadecuada de antibióticos entre $20 \%$ y $85 \%$ de los pacientes (8).

Numerosas han sido las estrategias para controlar la calidad de la prescripción de antimicrobianos para contener la resistencia bacteriana $(9,10)$. Tales estrategias abarcan actividades educativas dirigidas a los profesionales de la salud, consultas de las prescripciones con expertos, elaboración de guías de prescripción y auditorías de la calidad, entre otras intervenciones restrictivas y estructurales.

En general, los programas de control de antibióticos, resumidos por Davey y colaboradores (10), han mostrado su eficacia en cuanto a mejorar la calidad de la prescripción y reducir la resistencia microbiana en hospitales por medio de estrategias aisladas o en combinación. Igualmente importantes para el control de la resistencia son las medidas aplicadas en los hospitales para prevenir la transmisión de estos microorganismos al ambiente, a otros pacientes y a los trabajadores de la salud (11). En nuestra búsqueda, no encontramos trabajos publicados recientemente sobre programas de control de antibióticos en el ámbito hospitalario de Latinoamérica o Cuba.

En vista de la evidencia acerca de las deficiencias del empleo de antimicrobianos en Cuba, realizamos un estudio con el objetivo de mostrar la eficacia potencial de un programa de control en la calidad de la prescripción de antibióticos en un hospital de nivel secundario de La Habana, Cuba.

\section{MATERIALES Y MÉTODOS}

Se realizó un estudio de intervención en el Hospital Docente Clínico Quirúrgico Joaquín Albarrán en el período entre el 1 de mayo de 2008 y el 31 de marzo de 2011. Este hospital es una institución de nivel secundario del sistema de salud de La Habana; cuenta con 385 camas y ofrece servicios clínicos y quirúrgicos a alrededor de 300000 adultos de los municipios del oeste de la ciudad; también presta servicios de urgencias y ambulatorios.

\section{Programa de control de antibióticos}

La intervención realizada consistió en una auditoría de la calidad de la prescripción y la retroalimentación de la información, actividades educativas, funcionamiento de un comité de antibióticos y la elaboración de guías de uso de antimicrobianos.

\section{Auditoría de la calidad de la prescripción}

Un día de cada mes, elegido por conveniencia, se estudió el total de pacientes hospitalizados. De la historia clínica de aquellos que se encontraban utilizando antimicrobianos se obtuvieron datos sociodemográficos, información sobre la presencia de infección al ingreso y localización de la infección, antecedentes patológicos, procedimientos quirúrgicos, alergias a antibióticos, resultados de estudios microbiológicos, descripción de la administración de antimicrobianos (medicamento, dosis, intervalo, vía de administración y duración del tratamiento). Además, acerca de cada antimicrobiano utilizado, se registró si la indicación fue empírica, específica (basada en la microbiología) o para profilaxis perioperatoria. Finalmente, se registraron los exámenes complementarios que permitieron evaluar la función renal o hepática. La información anterior fue analizada con expertos en uso de antimicrobianos, quienes determinaron qué pacientes habían recibido una prescripción inadecuada, decisión que se fundamentó en los principios del uso de antimicrobianos y las políticas de los servicios $(12,13)$. Se consideraron causas de uso inadecuado las siguientes: 1) no indicado o innecesario, cuando el antibiótico se utilizó sin evidencia clínica de infección o justificación para su uso profiláctico; 2) dosis o intervalo incorrecto; 3 ) selección incorrecta, cuando se prescribió un antimicrobiano no apropiado para la infección del paciente o el fármaco seleccionado era más tóxico o se utilizó una combinación incorrecta o innecesaria, y 4) duración inapropiada del tratamiento.

\section{Retroalimentación de la información}

La información sobre la calidad de la prescripción, incluida la descripción de los pacientes cuya prescripción fue inadecuada, se envió a los servicios médicos mediante informes impresos y electrónicos, todos con frecuencia mensual.

\section{Actividades educativas}

Se realizó una distribución de trabajos publicados pertinentes a todos los profesionales responsables de prescribir antibióticos en el hospital por medio de una lista electrónica de distribución, en la cual se incluyó además una discusión de los casos seleccionados. Se impartieron cinco cursos y cuatro talleres a partir de septiembre 2008. Los cursos (básico y avanzado) versaron sobre el uso de antimicrobianos y el tratamiento de la neumonía y otras infecciones respiratorias agudas, mientras que en los talleres se trataron las prácticas de uso de antimicrobianos y prevención del uso inadecuado, políticas de uso hospitalario, profilaxis antibiótica perioperatoria y uso en cirugía general. Además, durante el período del estudio y con la participación de un miembro del comité de antibióticos, se realizó mensualmente el análisis de los errores de prescripción en los colectivos médicos.

\section{Comité de antibióticos}

A partir de agosto de 2008, un comité de antibióticos compuesto por licenciados en farmacia, infectólogos, cirujanos, especialistas en medicina interna y microbiólogos evaluaron en sesiones diarias (de lunes a viernes) las propuestas de pautas terapéuticas para los pacientes hospitalizados presentadas por los médicos asistenciales y fundamentadas en los elementos clínicos y los estudios complementarios.

\section{Elaboración de guías de uso de antimicrobianos}

Los servicios clínicos y quirúrgicos elaboraron guías de tratamiento antibiótico para las infecciones y para la profilaxis perioperatoria, tomando en consideración la mejor evidencia publicada y el cuadro básico de medicamentos. Las guías se pusieron en práctica a partir de agosto de 2008.

El estudio fue aprobado por el Comité de Ética de las Investigaciones del Hospital Joaquín Albarrán y del Instituto Superior de Ciencias Médicas de La Habana, y registrado como proyecto ramal de investigación del Ministerio de Salud Pública (http://www.isss.sld.cu/node/32). La información obtenida de la historia clínica, incluidos los datos de los pacientes (iniciales del nombre, número de la historia) y de los profesionales que emitieron las prescripciones (nombre, especialidad), fueron de conocimiento exclusivo del equipo de investigación y no se comuni- 
CUADRO 1. Características demográficas y clínicas de los pacientes estudiados. Hospital Joaquín Albarrán, La Habana, Cuba, mayo de 2008 a febrero 2011

\begin{tabular}{lcr}
\hline \multicolumn{1}{c}{ Característica } & No. & $\%$ \\
\hline Edad en años (media \pm DE) & $64,0(17,8)$ & \\
Sexo & 1538 & 53,4 \\
$\quad$ Masculino & 1343 & 46,6 \\
$\quad$ Femenino & 1197 & 41,5 \\
Antecedentes patológicos & 580 & 20,1 \\
$\quad$ Hipertensión arterial & 432 & 15,0 \\
Diabetes mellitus & 387 & 13,4 \\
Insuficiencia coronaria & 323 & 11,2 \\
Insuficiencia respiratoria & 85 & 2,9 \\
Cáncer & 37 & 1,3 \\
Insuficiencia renal crónica & 2215 & 77,1 \\
$\quad$ Insuficiencia hepática & & \\
Infección al ingreso & 1353 & 61,1 \\
Localización de la infección ${ }^{a}$ & 254 & 11,5 \\
$\quad$ Tracto respiratoria & 223 & 10,1 \\
Vías urinarias & 367 & 16,6 \\
Piel y partes blandas & & \\
Otra & 600 & 20,8 \\
Procedimiento quirúrgico durante & & \\
la hospitalización &
\end{tabular}

caron a terceros. Los registros primarios y las bases de datos se mantuvieron protegidos en el Departamento de Epidemiología del Hospital Joaquín Albarrán.

\section{Procesamiento y análisis}

Con los resultados de las auditorías se calculó la proporción de uso inadecuado de antimicrobianos (pacientes en los que se determinó que habían recibido prescripción inadecuada dividido por el total de pacientes estudiados expresados por 100 pacientes). Se construyó un gráfico aritmético simple de la serie temporal y se compararon los valores absolutos de las proporciones de la serie entre sí (figura 1). Para determinar que efectivamente había cambios en la tendencia de la serie, se utilizó un modelo de regresión lineal simple, para el que se utilizó el paquete estadístico EViews 5.0.

\section{RESULTADOS}

Se analizaron las historias clínicas y las prescripciones de antibióticos de 2941 pacientes, cuya edad promedio fue de 64 años (desviación estándar 17,8 años). $\mathrm{El}$ antecedente patológico más frecuente fue la hipertensión arterial $(41,5 \%)$, seguida en orden descendente por la diabetes mellitus $(20,1 \%)$ y la insuficiencia coronaria $(15,0 \%)$. Del total de pacientes, $77,1 \%$ presentaban infecciones al ingreso al hospital, predominantemente del aparato respiratorio $(61,1 \%)$, y con menor frecuencia, infecciones urinarias y de piel y partes blandas. Fue necesario practicar procedimientos quirúrgicos a 600 pacientes $(20,8 \%)$.

La figura 1 muestra la serie mensual de los valores de la proporción de uso inadecuado de antimicrobianos en el Hospital Joaquín Albarrán en el período comprendido entre 1 de mayo de 2008 y el 31 de marzo de 2011. En la figura se observa que el uso inadecuado mostró en los primeros tres meses del período estudiado valores más altos y alcanzó su máximo de $48,8 \%$ en julio de 2008; es decir, durante el período en el cual las medidas de modificación de las prácticas no habían alcanzado su mayor eficacia. Posteriormente se observa fluctuación en la serie de datos con una tendencia a la disminución de la proporción de uso inadecuado que oscila alrededor de $20 \%$. Con respecto al modelo lineal de tendencia ajustado, se encontró que el valor tomado por la pendiente, aunque se encontraba cercano a cero, era negativo y significativamente diferente de cero $(\beta=-0,29 ; P=0,02)$.

\section{DISCUSIÓN}

Los antibióticos constituyen unos de los grupos farmacológicos más utilizados en la práctica clínica y generan un

FIGURA 1. Proporción de uso inadecuado de antimicrobianos (por 100 pacientes), hospital Joaquín Albarrán, mayo de 2008 a marzo de 2011 , por mes

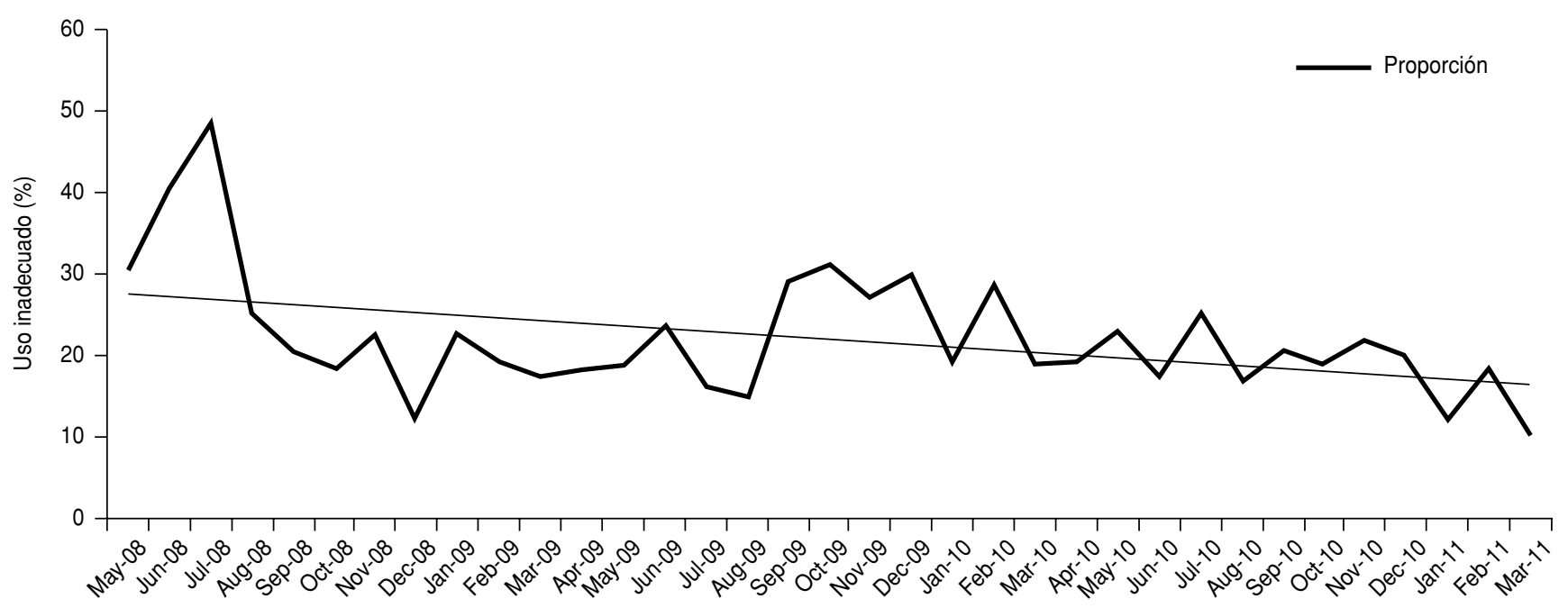


gasto importante a los sistemas de salud, incluido su efecto en la calidad de los servicios sanitarios relacionado con el incremento de la morbilidad por infecciones y de la mortalidad, a lo que su uso inadecuado contribuye de forma significativa $(1-3,14)$. Wirtz y colaboradores muestran la tendencia del uso de antibióticos en países de América Latina en la cual se observan diferencias entre países y la necesidad de emplazar políticas que conduzcan a mejorar la calidad de la prescripción (15). Por otra parte, Ranji y colaboradores (5) realizaron un análisis crítico de investigaciones publicadas sobre estrategias de control de la calidad de la prescripción de antibióticos. Observaron que en 24 ensayos clínicos hubo una reducción neta de la tasa de uso de antimicrobianos de hasta $29,5 \%$, la que fue en promedio de $8,9 \%$. De esos ensayos clínicos, $50 \%$ utilizaron estrategias múltiples con actividades educativas, auditoría y retroalimentación de la información a quienes recetaban los antibióticos. En general, estos autores muestran que las medidas de control son valiosas para reducir la frecuencia del uso de antibióticos y de su prescripción inadecuada. Davey informa resultados similares (10).

La utilización de comités multidisciplinarios de antibióticos o especialistas en enfermedades infecciosas y la retroalimentación y capacitación de quienes prescriben han sido las medidas más utilizadas, según los estudios publicados. Estas prácticas han mostrado su eficacia cuando se trata de reducir las tasas de consumo, las prescripciones inadecuadas y la resistencia microbiana en los hospitales (16-20). Por ejemplo, Raineri informa un incremento de $20,4 \%$ en la adherencia a las guías locales de prescripción de antibióticos y disminución de la mortalidad hospitalaria mediante la consulta con especialistas en enfermedades infecciosas (18).

El programa de control de antibióticos que se implemente debe tener como característica esencial el empleo simultáneo de estrategias persuasivas y restrictivas, incluidas las estrategias de control recomendadas por la Sociedad Estadounidense de Enfermedades Infecciosas (21). De las medidas persuasivas, se deben destacar las siguientes: 1) El análisis de la prescripción inadecuada identificada en las auditorías con los colectivos médicos; 2) la toma de la mejor decisión para la prescripción de un antibiótico, colegiada en el comité de antibióticos, y 3) las actividades educativas que utilizan una combinación de estrategias activas y pasivas, ya que uno de los elementos sólidamente relacionados con la prescripción inade- cuada es la deficiencia de conocimientos sobre el uso de antibióticos.

En el análisis de los resultados del presente trabajo, se debe tener en cuenta que este no fue un estudio controlado y no puede determinarse la importancia relativa de cada medida de intervención en la modificación de las prácticas de prevención. Asimismo la inclusión de otros indicadores de impacto relacionados con la resistencia microbiana y el consumo de antimicrobianos podría brindar información de valor para apoyar la evidencia relacionada con el cambio en los indicadores de calidad. Es posible poner en marcha programas similares a este en instituciones hospitalarias si se tienen en cuenta las particularidades del hospital en cuestión (recursos humanos, complejidad de sus servicios) con el fin de priorizar los componentes a aplicar.

El presente estudio es el primero realizado en Cuba sobre este tema y ha mostrado que el programa de control de antibióticos mejora la calidad de la prescripción para los pacientes hospitalizados. Es recomendable su consolidación mediante la introducción de otras estrategias válidas, como el ciclado antimicrobiano y el desescalado terapéutico, además de la evaluación de su impacto en el consumo de antibióticos y en la resistencia antimicrobiana.

\section{REFERENCIAS}

1. Estrategia mundial de la OMS para contener la resistencia a los antimicrobianos. Organización Mundial de la Salud. 2001. WHO/CDS/ CSR/2001.2

2. Livermore DM. Bacterial resistance: origins, epidemiology and impact. Clin Infect Dis. 2003:36(suppl 1):511-23.

3. Sande Broinsma N, Grudmann H, Verloo D, Tiemersma E, Monen J, Goosens H, et al. Antimicrobial drugs use and resistance in Europe. Emerg Infect Dis 2008;14(11):1722-30.

4. Martinez JS, Le Falher G, Corne P, Bourdin A, Lequellec A, Delabre JP, et al. Adherence to antibiotherapy guidelines for acute communityacquired pneumonia in adults, in a teaching hospital. Med Mal Infect. 2010;40(8):468-75.

5. Tourmousoglou CE, Yiannakopoubo E Ch, Kalapothaki U, Bramis J, Papadopoulos JST. Adherence to guidelines for antibiotic prophylaxis in general surgery: a critical appraisal. J Antimicrob Chemoth. 2008;61(1):214-8.

6. Apisarnthanarak A, Danchaivijitr S, Bailey TC, Fraser VT. Inappropiate use of antibiotic in a terciary care center in Thailand: a prevalence study and review of experience in Thailand. Infect Control Hosp Epidemiol. 2006;27(4):416-20.

7. Guanche $H$, Izquierdo-Cubas $F$, Zambrano $A$, Frómeta I, Bastanzuri M, Malpica J, et al. Uso de antimicrobianos en Instituciones de salud de Cuba. Medicrit. 2009;6(1):24-30.

8. Mir Narbona I, Guanche Garcell H, Chappi Estévez Y, Díaz Piñera A, Rodríguez Uribe S, Fiterre Lancis I, et al. Calidad de prescripción de antimicrobianos en servicios seleccionados en hospitales clínico quirúrgicos. Archivos Venezolanos de Farmacología y Terapéutica. 2009;28(2):63-6.

9. Ranji SR, Steinman MA, Shojania KG, Sundaram V, Lewis R, Arnold S, et al. Antibiotic prescribing behavior. Vol. 4 of: Shojania KG, McDonald KM, Wachter RM, Owens DK, editors. Closing the Quality Gap: A Critical Analysis of Quality Improvement Strategies. Technical Review 9 (Prepared by the Stanford University-UCSF Evidence-based Practice Center under Contract No. 290-02-0017). AHRQ Publication No. 04(06)-0051-4. Rockville, MD: Agency for Healthcare Research and Quality. January 2006.
10. Davey P, Brown E, Fenelon L, Finch R, Gould I, Hartman G, et al. Interventions to improve antibiotic prescribing practices for hospital inpatients. Cochrane Database of Systematic Reviews 2005, Issue 4. Art. No.: CD003543. DOI: 10.1002/14651858.CD003543.pub2.

11. Centers for Disease Control and Prevention, Healthcare Infection Control Practices Advisory Committee. Management of multidrugresistant organisms in healthcare settings, 2006. Atlanta, GA: US Department of Health and Human Services, CDC, Healthcare Infection Control Practices Advisory Committee; 2007. Disponible en: http://www.cdc.gov/ ncidod/dhqp/pdf/ar/mdroguideline2006. pdf Acceso el 15 de marzo 2011.

12. Levison ME. Bacterias y fármacos antimicrobianos. En: Beers M H, Editor. El Manual Merck de diagnóstico y tratamiento. 11 Edición. Madrid: Elsievier; 2007. Pp. 1537-71.

13. Leekha S, Terrell CL, Edson RS. General principles of antimicrobial therapy. Mayo Clin Proc. 2011;86(2):156-67.

14. Cisneros JM, Ortiz Leyva C, Lepe JA, Obando I, Conde M, Cayuela A, et al. Uso prudente 
de antibióticos y propuestas de mejora desde la medicina hospitalaria. Enferm Infecc Microbiol Clin. 2010;28(suppl 4):28-31.

15. Wirtz VI, Dreser A, Gonzales R. Trends in antibiotic utilization in eight Latin American countries 1997-2007. Rev Panam Salud Publica. 2010;27(3):219-25.

16. Camins BC, King MD, Wells JB, Googe HL, Patel M, Kourbatova EV, et al. Impact of an antimicrobial utilization program on antimicrobial use at a large teaching hospital: a randomized controlled trial. Infect Control Hosp Epidemiol. 2009;30:931-8.

17. Arnold FW, Mc Donald LC, Scott Smit R, Newman D, Ramirez JA. Improving antimicrobial use in the hospital setting by providing usage feedback to prescribing physicians. Infect Control Hosp Epidemiol. 2006;27:378-82.
18. Raineri E, Pan A, Mondello P, Acquarolo A, Candiani A, Crema L. Role of the infectious diseases specialist consultant on the appropriateness of antimicrobial therapy prescription in an intensive care unit. Am J Infect Control. 2008;36(4):283-90.

19. Aldeyab MA, Monner DL, Lopez Lozano JM, Hughes CM, Scott MG, Kearney MP, et al. Modelling the impact of antibiotic use and infection control practices on the incidence of hospital acquired methicillin resistance Staphylococcus aureus: a time series analysis. J Antim Chemother. 2008;62:593-600.

20. Miliani K, L'Heriteau F, Afandari S, Arnaud I, Costa Y, Deliere E, et al. Specific control measures for antibiotic prescription are related to lower consumption in hospitals: results from a French multicenter pilot study. J Antim Chemother. 2008;62:823-829.

21. Dellit TH, Owens RC, Mc Gowan J, Gerding DN, Weinstein RA, Burke JP, et al. Infectious Disease Society of America and the Society for Healthcare Epidemiology of America. Guidelines for developing an institutional program to enhance antimicrobial stewardship. Clin Infect Dis. 2007;44:159-77.

Manuscrito recibido el 14 de abril de 2011. Aceptado para publicación, tras revisión, el 22 de noviembre de 2011.

ABSTRACT Objective. Demonstrate the efficacy of a quality control program on antibiotic prescription in Joaquín Albarrán Hospital in Havana, Cuba.

Methods. An interventional study was conducted from 1 May 2008 to 31 March

Impact of a quality control program on antibiotic prescription in a hospital in Havana, Cuba quality, information feedback educational activities, the operations of an antibiotic committee, and the preparation of protocols on antimicrobial drug use. A simple arithmetic graph of the time series was constructed, and the absolute values of the series percentages were compared. In order to verify the existence of a series trend, a simple linear regression model was applied.

Results. Antibiotic prescription was evaluated in 2941 patients. An irregular series was observed, with inappropriate use in $30.7 \%-48.4 \%$ of these patients in the first three months analyzed. The value of the regression slope was close to zero, although it was negative and significantly different from zero $(\beta=-0.29 ; P=0.02)$.

Conclusions. The antibiotic control program improved the quality of prescribing for hospital patients.

Key words Drug prescriptions; drug and narcotic control; anti-bacterial agents; Cuba. 